\title{
BRIOFITAS SOBRE CARPOFOROS DE HONGOS
}

\section{BRYOPHYTES ON CARPOPHORES OF FUNGI}

\author{
Victor Ardiles Huerta ${ }^{1}$, Felipe Osorio-Zúñiga ${ }^{2} \&$ Elizabeth Barrera Moscoso $^{1}$
}

\begin{abstract}
${ }^{1}$ Museo Nacional de Historia Natural, Sección Botánica, Casilla 787, Santiago, Chile; ${ }^{2}$ Universidad Austral de Chile, Facultad de Ciencias, Instituto de Ecología y Evolución, Casilla 567, Valdivia, Chile.

vittoko2002@yahoo.es
\end{abstract}

\begin{abstract}
RESUMEN
Se registra el desarrollo de briófitas sobre carpóforos de hongos de estructura coriácea, leñosa y perenne. Los resultados revelan la presencia de siete especies de hepáticas foliosas: Bazzania peruviana, Heteroscyphus integrifolius, Leiomitria elegans, Lepidozia chordulifera, Plagiochila bispinosa, Porella chilensis, Telaranea blepharostoma y dos musgos: Dicranoloma robustum e Hypnum chrysogaster. Se propone que los hongos de estas características son un apropiado sustrato para el desarrollo de algunas especies de hepáticas foliosas y musgos. Este artículo es una primera aproximación al estudio de briófitas que crecen sobre la superficie de carpóforos de hongos.
\end{abstract}

PALABRAS ClAVE: Hepáticas, musgos, estructura coriácea.

\section{ABSTRACT}

The development of bryophytes is registered on carpophores of fungi coriaceous, woody and perennial structure. The results reveal the presence of seven foliose liverworts: Bazzania peruviana, Heteroscyphus integrifolius, Leiomitria elegans, Lepidozia chordulifera, Plagiochila bispinosa, Porella chilensis, Telaranea blepharostoma and two mosses: Dicranoloma robustum and Hypnum chrysogaster. We propose that the fungi of these characteristics are an appropriate substratum for the development of some species of foliose liverwort and mosses. This article is the first approximation to the study of bryophytes that grow on the surface of fungi carpophore.

KEYwORDS: Liverworts, mosses, coriaceous structure.

\section{INTRODUCCION}

En general la literatura sostiene que las briófitas pueden desarrollarse sobre superficies como rocas, grietas, corteza, madera en descomposición, estiércol, cadáveres de animales, cuero, cutículas de hojas, techumbres y concreto (Smith 1982, Schofield 2001, Bates 2000), sin embargo durante un trabajo de campo realizado en un pequeño parche de Bosque Valdiviano, en la Cordillera de la Costa de la Provincia de Valdivia, se encontraron briófitas sobre la superficie de carpóforos de estructura coriácea, leñosa y perenne.

En sentido estricto, el carpóforo representa el cuerpo fructífero o la porción visible del hongo, el cual posee diferentes formas y tamaños, siendo en general efímero y caduco. No obstante, unas pocas especies poseen carpóforos coriáceos, leñosos y perennes. Este grupo de hongos son frecuentemente saprótrofos, lignícolas y a veces parásitos de árboles debilitados o heridos (Font Quer 2000, Lazo 2001, Llimona 1997, Ulloa \& Hanlin 2000).

El objetivo de este artículo es determinar las especies de briófitas que se registraron sobre los carpóforos de los hongos recolectados, como también discutir brevemente esta interesante relación ecológica entre plantas criptógamas y hongos. 


\section{MATERIALES Y METODOS}

En Febrero del 2007 se recolectaron carpóforos en un pequeño parche de Bosque Valdiviano, en la Cordillera de la Costa, Provincia de Valdivia, Región de Los Ríos, Chile (3942'52”'S; 73¹9'50”'W; 470 m s.n.m.). Dicho parche de bosque posee una vegetación mixta de Laureliopsis philippiana (Molina) Molina, Weinmannia trichosperma Cav., Saxegothaea conspicua Lindl. y Drimys winteri J.R.Forst. et G.Forst. (Lepez 1998).

Tres carpóforos fueron recolectados desde la corteza de un ejemplar de Saxegothaea conspicua y cuatro desde la superficie de un ejemplar caído de Drimys winteri. Para cada carpóforo estudiado se hizo un análisis macro y micromorfológico, utilizando el microscopio óptico y microscopio estereoscópico. Para la realización del análisis microscópico se efectuaron cortes de las distintas zonas del carpóforo, a mano alzada con bisturí. Las referencias consultadas para la identificación fueron Espinosa (1921), Kreisel (1971) y Lazo (2001). La nomenclatura siguió el trabajo de Minter (2006). Para la verificación de las especies se comparó con ejemplares de herbario (SGO).

La identidad de las briófitas se obtuvo sobre la base de análisis macro y micromorfológico. Las referencias consultadas para la identificación fueron
Buck (2002), Engel (1978), Gradstein \& Da Costa (2003), Larraín (2007) y Schuster (2000). La nomenclatura para los musgos siguió la propuesta por He (1998), y para las hepáticas la de Hässel de Menéndez \& Solari (1985). Para la verificación de las especies se comparó con ejemplares de herbario (SGO). Todo el material estudiado (Tabla I) está depositado en el Herbario del Museo Nacional de Historia Natural (SGO).

\section{RESULTADOS}

Fueron analizados un total de siete carpóforos, tres de los cuales corresponden a Phellinus senex (Nees et Mont.) Imazeki y cuatro a Trichaptum sector (Ehrenberg) Kreisel. Phellinus senex posee un carpóforo aplanado o convexo unguliforme, solitario o agrupado, sésil, duro, con porciones delgadas muy quebradizas, color castaño o castañoacanelado o mohoso, con crestas concéntricas tuberculosas. La superficie inferior es aterciopelada al tacto, con setas himeniales cónicas, de paredes gruesas. Habitan huecos de troncos vivos de Saxegothaea conspicua, algunas Mirtáceas y sobre troncos muertos. Se distribuye en América del Sur, América Central, India, Madagascar, Filipinas (Espinosa 1921, Fig. 1).

TABLA I. Listado de briófitas registradas sobre los carpóforos de Phellinus senex y Trichaptum sector $(\mathrm{X}=$ presencia), y materiales estudiados.

TABLE I. Checklist of bryophytes recorded on carpophore of Phellinus senex and Trichaptum sector ( X = presence), and studied materials.

\begin{tabular}{|c|c|c|c|c|c|c|c|}
\hline & \multicolumn{4}{|c|}{ Trichaptum sector } & \multicolumn{3}{|c|}{ Phellinus senex } \\
\hline & SGO & SGO & SGO & SGO & SGO & SGO & SGO \\
\hline HEPATICAS & 155323 & 155322 & 155325 & 155324 & 155329 & 155328 & 155327 \\
\hline Bazzania peruviana & & & & & $\mathrm{X}$ & $\mathrm{X}$ & $\mathrm{X}$ \\
\hline Heteroscyphus integrifolius & & & & & $\mathrm{X}$ & $X$ & $X$ \\
\hline Leiomitria elegans & $\mathrm{X}$ & $\mathrm{X}$ & $\mathrm{X}$ & & & $\mathrm{X}$ & \\
\hline Lepidozia chordulifera & $\mathrm{X}$ & $\mathrm{X}$ & $\mathrm{X}$ & $\mathrm{X}$ & $X$ & & $\mathrm{X}$ \\
\hline Plagiochila bispinosa & $\mathrm{X}$ & $\mathrm{X}$ & $\mathrm{X}$ & $\mathrm{X}$ & & & \\
\hline Porella chilensis & & & & $\mathrm{X}$ & & & \\
\hline Telaranea blepharostoma & $X$ & & & & & $X$ & $X$ \\
\hline MUSGOS & & & & & & & \\
\hline Dicranoloma robustum & & & & & & $\mathrm{X}$ & \\
\hline Hypnum chrysogaster & & & & & $X$ & & $\mathrm{X}$ \\
\hline
\end{tabular}


Trychaptum sector tiene un carpóforo crustáceo, dentiforme, con trama parda de estructura doble, arriba floja y tomentosa, abajo más sólida; superficie del píleo cubierta de pelos largos, ramificados. Esporóforos escaleriformes muy extendidos. Habita troncos y ramas caídas de árboles de América tropical y subtropical (Kreisel 1971, Fig. 2).

Se registró un total de nueve especies de briófitas creciendo sobre la superficie de los carpóforos, de las cuales siete son hepáticas foliosas; Bazzania peruviana (Ness) Trevis, Heteroscyphus integrifolius (Lehm. \& Lindenb.) Fulf., Leiomitria elegans (Lehm. ex De Not.) Hässel, Lepidozia chordulifera Taylor, Plagiochila bispinosa Lindenb. ex Gottsche, Porella chilensis (Lehm. \& Lindenb.)
Trevis, Telaranea blepharostoma (Steph.) Fulford y 2 son musgos, Dicranoloma robustum (Hook.f. \& Wilson) Paris y Hypnum chrysogaster Müll.Hal. No se registró ningún antocerote (Tabla I).

Las hepáticas Bazzania peruviana (Fig. 3), Heteroscyphus integrifolius (Fig. 4) se encontraron en los carpóforos de Phellinus senex. Plagiochila bispinosa (Fig. 5) y Porella chilensis se hallaron en los carpóforos de Trychaptum sector. Lepidozia chordulifera, Leiomitria elegans (Fig. 6) y Telaranea blepharostoma fueron halladas sobre los carpóforos de ambas especies de hongos. Los musgos Dicranoloma robustum e Hypnum chrysogaster (Fig. 7) se encontraron sólo sobre los carpóforos de Phellinus senex (Tabla I).

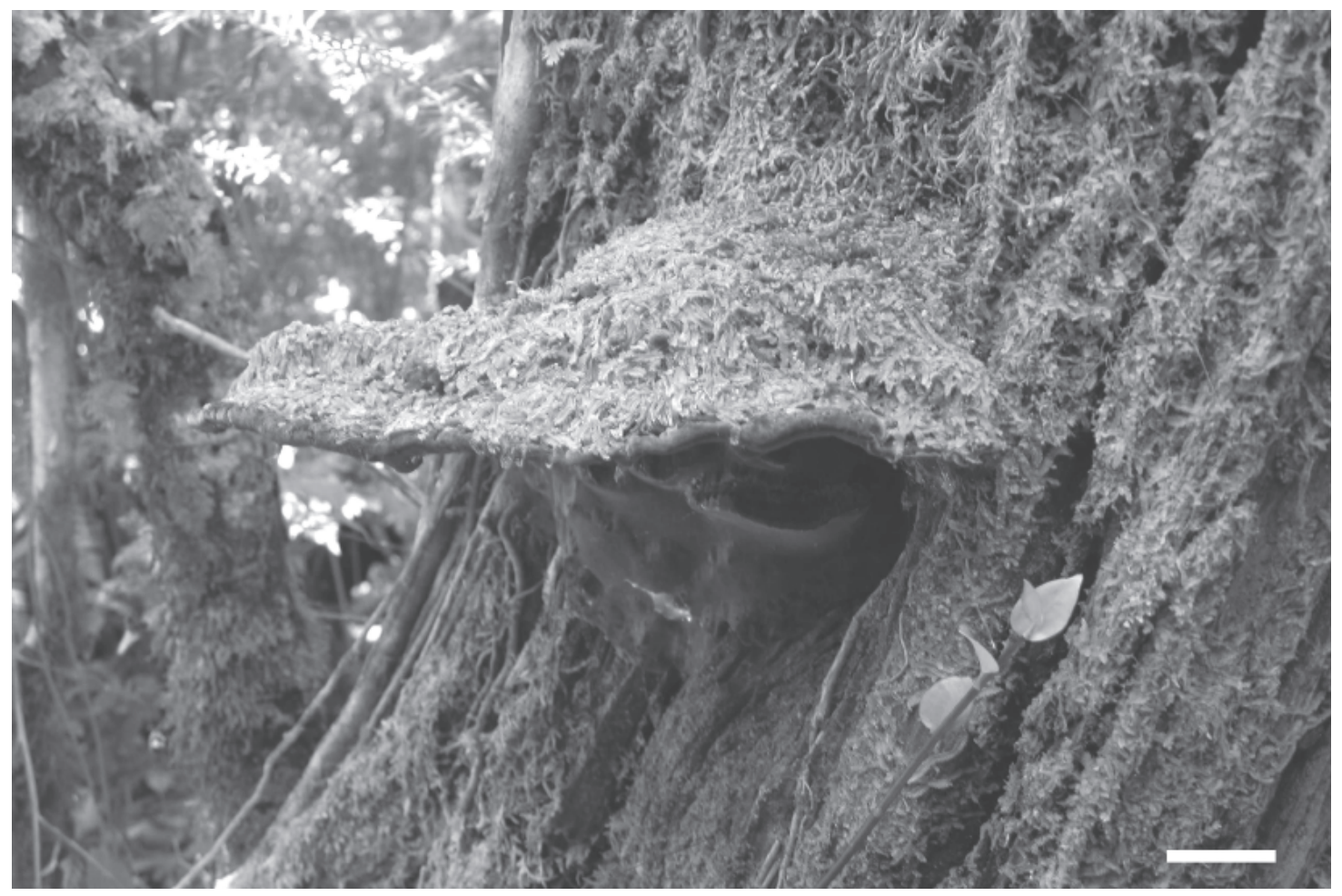

Figura 1. Carpóforo de Phellinus senex sobre el tronco de Saxegothaea conspicua $($ Escala $=2 \mathrm{~cm})$.

FIgURE 1. Carpophores of Phellinus senex on a trunk of Saxegothaea conspicua $(\mathrm{Scale}=2 \mathrm{~cm})$. 


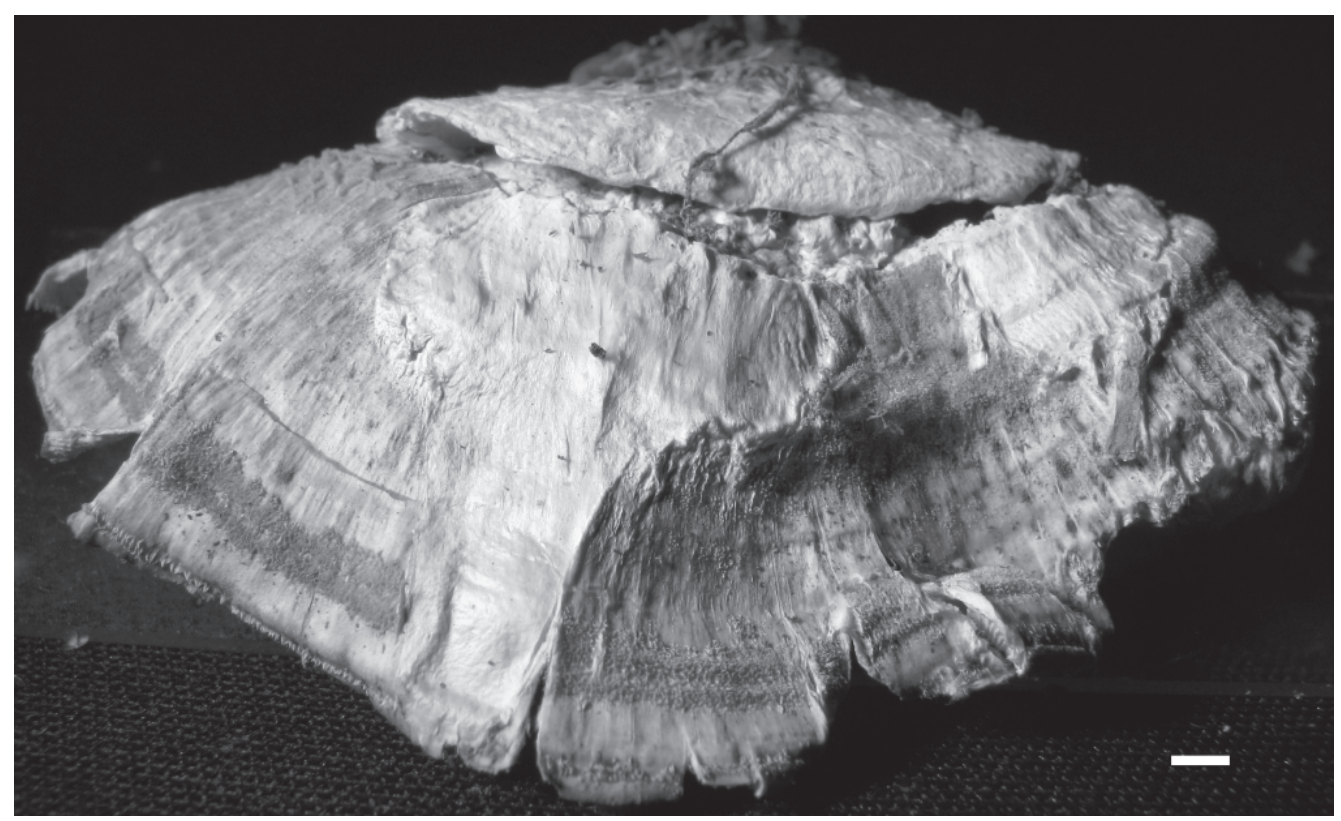

FiguRA 2. Vista general de un carpóforo de Trichaptum sector (Escala $=5 \mathrm{~mm}$ ).

FIGURE 2. General view of a carpophore of Trichaptum sector (Scale $=5 \mathrm{~mm}$ ).

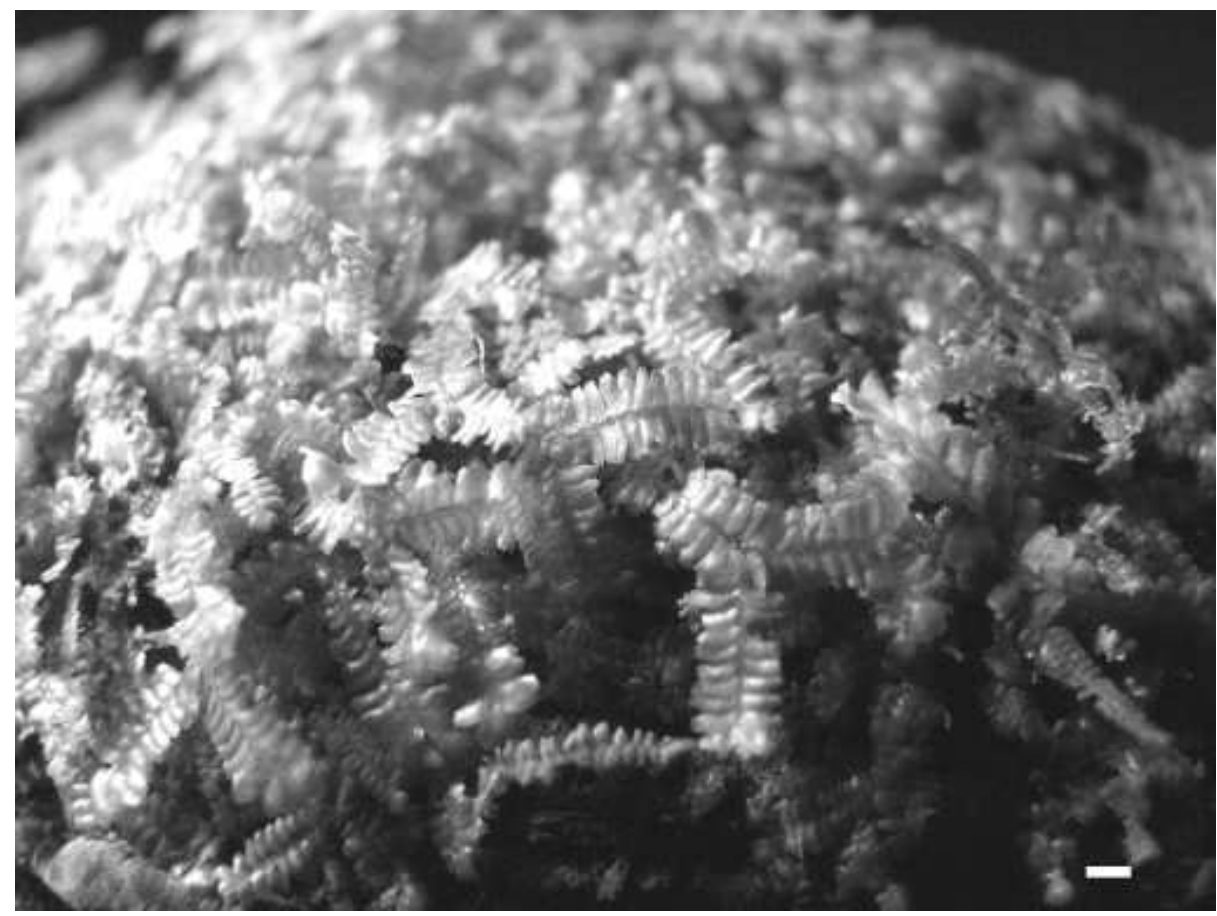

FIGURA 3. Gametofitos de Bazzania peruviana sobre la superficie de un carpóforo de Phellinus senex (Escala $=1 \mathrm{~mm}$ ). FIGURE 3. Gametophytes of Bazzania peruviana on surface of a carpophore of Phellinus senex (Scale =1 mm). 
Gayana Bot. 66(1), 2009

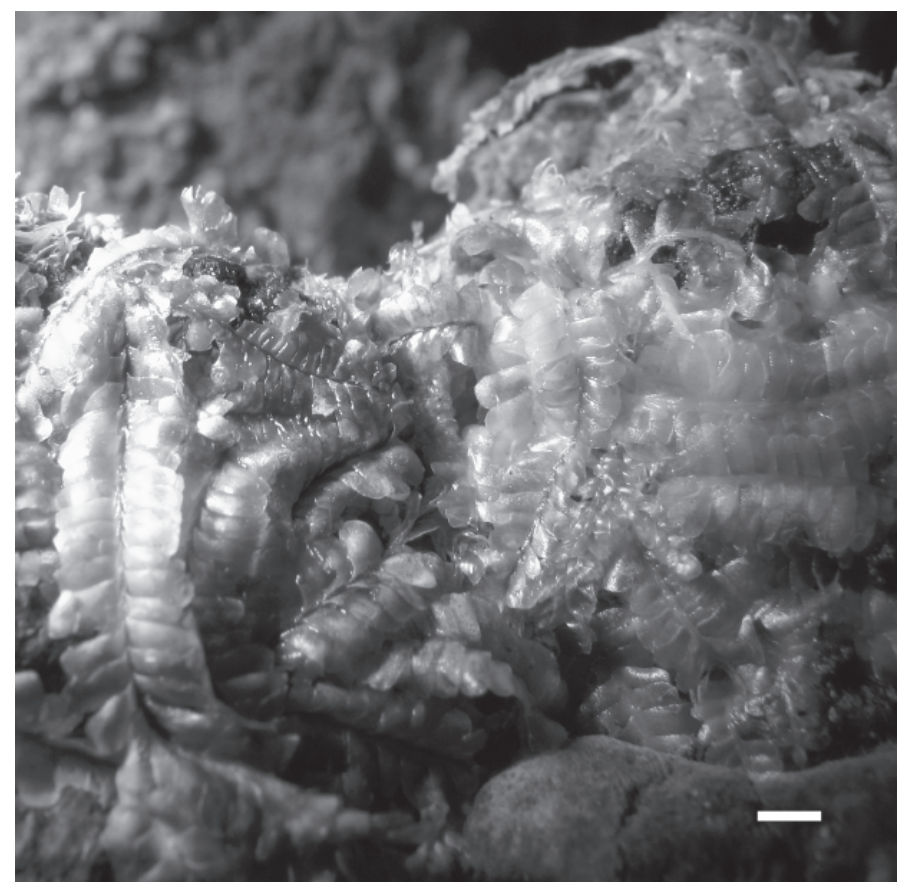

FIgURA. 4. Gametofitos de Heteroscyphus integrifolius sobre un carpóforo de Phellinus senex (Escala = $3 \mathrm{~mm}$ ).

FIGURE 4. Gametophytes of Heteroscyphus integrifolius on a carpophore of Phellinus senex (Scale $=3 \mathrm{~mm})$.

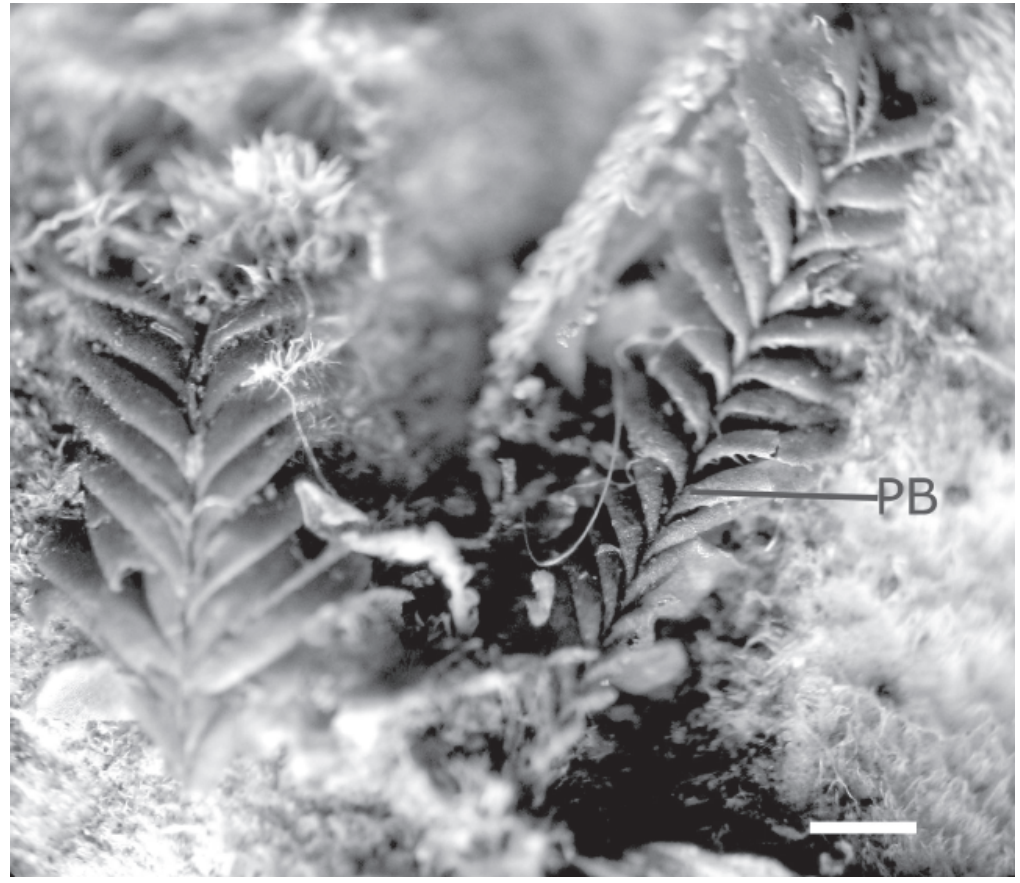

FiguRA 5. Gametofito de Plagiochila bispinosa $(\mathrm{PB})$ sobre la superficie de un carpóforo de Trychaptum sector $($ Escala $=1 \mathrm{~mm})$. FIGURA 5. Gametophyte of Plagiochila bispinosa $(\mathrm{PB})$ on the surface of a carpophore of Trychaptum sector $(\mathrm{Scale}=1 \mathrm{~mm})$. 
Briófitas sobre carpóforos de hongos: ARDILES, V. ET AL.

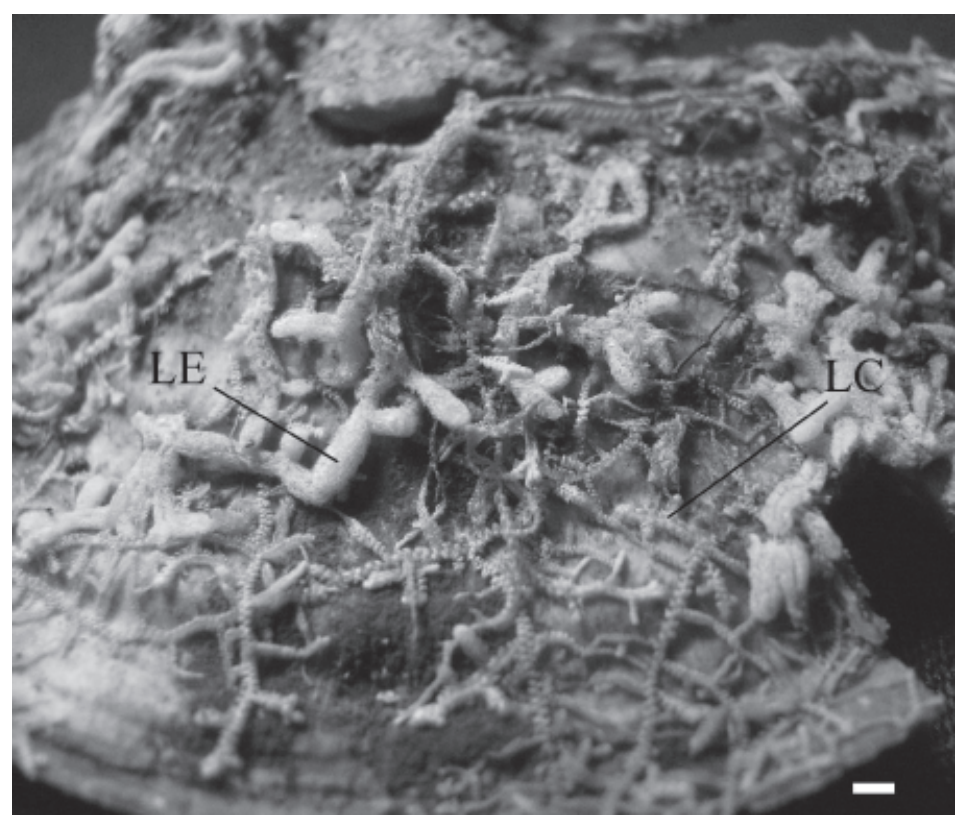

FIGURA 6. Gametófitos de Leiomitria elegans (LE) y Lepidozia chordulifera (LC) sobre la superficie de un carpóforo de Trychaptum sector $($ Escala $=1 \mathrm{~mm})$.

FIGURE 6. Gametophytes of Leiomitria elegans (LE) and Lepidozia chordulifera (LC) on the surface of a carpophore of Trychaptum sector $($ Scale $=1 \mathrm{~mm})$.

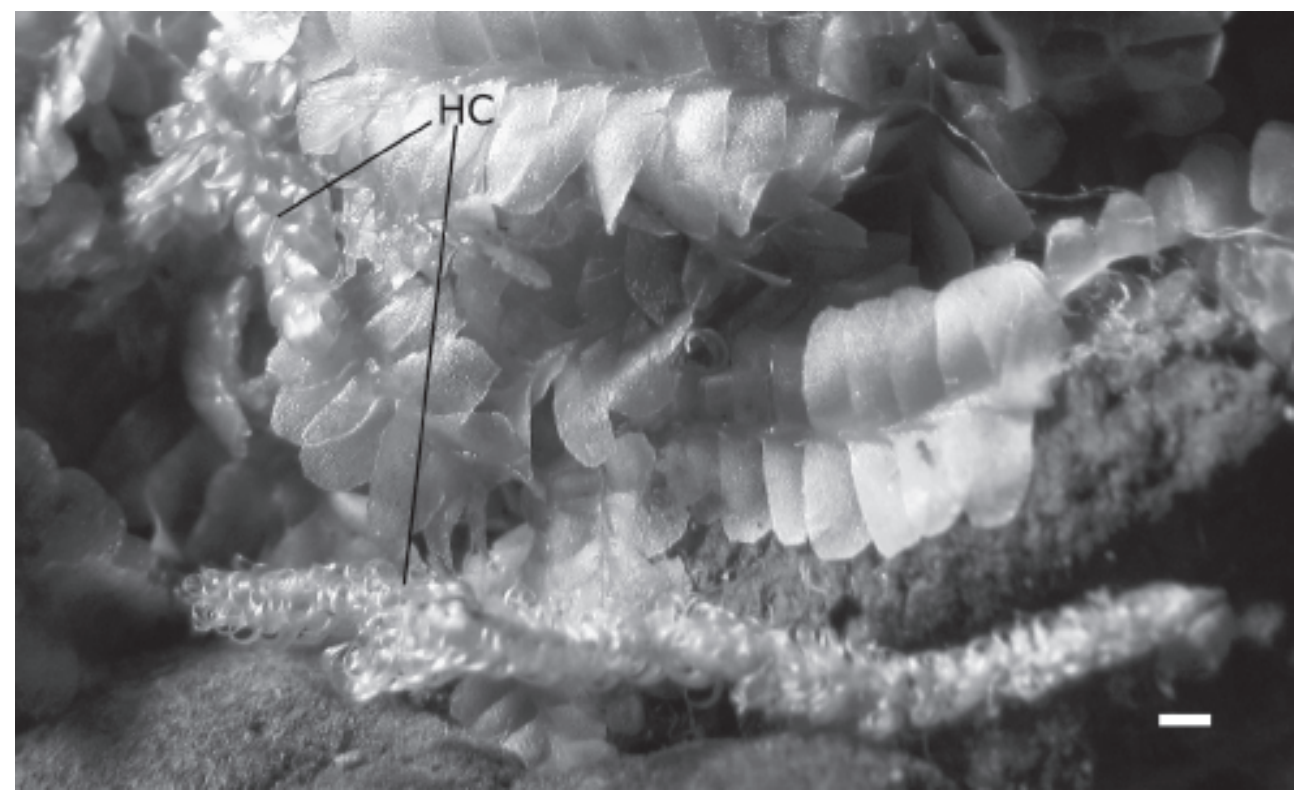

FiguRA 7. Gametofitos del musgo Hypnum chrysogaster $(\mathrm{HC})$ sobre la superficie de un carpóforo de Phellinus senex (Escala=1 mm). FigURA 7. Moss gametophyte of Hypnum chrysogaster $(\mathrm{HC})$ on the surface of a carpophore of Phellinus senex $(\mathrm{Scale}=1 \mathrm{~mm})$. 


\section{DISCUSION}

Los antecedentes publicados sobre las relaciones entre briofitas y hongos han sido fundamentalmente explorados desde la asociación saprobionta, micorrízica y comensalista del hongo con estructuras vegetativas o reproductivas, como hojas, rizoides y esporofitos de algunas especies de musgos y hepáticas (Currah \& Davey 2006, Davis et al. 2003, Döbbeler 1997, Jumpponen et al. 2003). En este artículo por primera vez se plantea la relación entre briófitas y una estructura fúngica reproductiva como es el carpóforo, a modo de sustrato para su desarrollo.

Varios autores han estudiado las especies que conforman las comunidades de briófitas de los bosques templados lluviosos del sur de Chile, encontrando similitudes en la brioflora que habita las cortezas de árboles y troncos caídos (Frahm 2002, Schaumann 2005, Villagrán et al. 2002, 2005). En este estudio, también se encontró especies de briófitos que además de crecer en estos sustratos, estan presentes sobre los carpóforos recolectados, como las hepáticas foliosas Bazzania peruviana, Leiomitria elegans, Lepidozia chordulifera, Plagiochila bispinosa, Telaranea blepharostoma y musgos del género Dicranoloma.

Interesante es el hecho que solo las hepáticas Lepidozia chordulifera, Leiomitria elegans y Telaranea blepharostoma (Tabla I) están presentes en ambos tipos de carpóforos recolectados. Las demás especies registradas están presentes sólo en un tipo de carpóforo, lo cual nos sugiere una cierta preferencia. No obstante, el bajo número de ejemplares recolectados no es suficiente para afirmar este supuesto, siendo necesario efectuar un mayor esfuerzo de muestreo en futuros trabajos para confirmar este hecho.

Estos primeros antecedentes conjeturan interesantes preguntas sobre las especies de briófitas que crecen asociadas a estos carpóforos: ¿Cuál es la sucesión de las especies al momento de colonizar estos carpóforos? ¿Todos los hongos con carpóforos de estas características permiten el desarrollo de briófitas en sus superficies?

Destacamos el presente artículo como una primera aproximación al conocimiento de las especies de hepáticas foliosas y musgos que pueden desarrollarse sobre los carpóforos de hongos de estructura coriácea, leñosa y perenne.

\section{AGRADECIMIENTOS}

A Parque Oncol y Pablo Lepez por el apoyo logístico en las labores de terreno. A Daniel Stanton por su ayuda con la literatura citada. A Pablo Sandoval por sus acertadas sugerencias micológicas y a la Sra. Mélica Muñoz por sus valiosos comentarios.

\section{BIBLIOGRAFIA}

BATES, J.W. 2000. Mineral nutrition, substratum ecology, and pollution. In: A.J. Shaw and B. Goffinet(eds.), Bryophyte Biology. 248-299. Cambridge University Press.

Buck, W.R. 2002. Preliminary key to the mosses of Isla Navarino, Chile (Prov. Antártica Chilena). Published by the author, The New York Botanical Garden, Bronx, NY 10458-5126, U.S.A., 147 pp.

Currah, R.S. \& M.L. Davey. 2006. Interactions between mosses (Bryophyta) and fungi. Canadian Journal of Botany 84: 1509-1519.

Davis, E.C., J.B. Franklin, A.J. Shaw \& R. Vilgalys. 2003. Endophytic Xylaria (Xylariaceae) among liverworts and angiosperms: Phylogenetics, distribution, and symbiosis. American Journal of Botany 90: 1661-1667.

DÖBBELER, P. 1997. Biodiversity of bryophylous ascomicetes. Biodiversity and Conservation 6: 721-738.

Engel, J. J. 1978. A Taxonomic and Phytogeographic Study of Brunswick Peninsula (Strait of Magellan). Hepaticae and Anthocerotae. Fieldiana Botany 41: $1-352$.

EsPinosA, M. 1921. Sobre las especies chilenas del género Fomes. Revista Chilena de Historia Natural 25: 321-343.

Font Quer, P. 2000. Diccionario de botánica. Segunda Edición. Ediciones Península, Barcelona, 1244 pp.

FRAHM, J.P. 2002. Ecology of bryophytes along altitudinal and latitudinal gradients in Chile. Studies in austral temperate rain forest bryophytes 16 . Tropical Bryology 21: 67-79.

Gradstein, S. R. \& D. P. da Costa. 2003. The Hepaticae and Anthocerotae of Brazil. Memoirs of The New York Botanical Garden 87: 1-318.

Hässel de MenÉndez, G.G. \& S. Solari. 1985. Catálogo de las hepáticas. En: O. Boelcke, D.M. Moore \& F.A. Roig (eds.), Transecta Botánica de la Patagonia Austral. 299-342. Conicet, Buenos Aires, Argentina.

HE, S. 1998. A Checklist of the mosses of Chile. Journal of the Hattori Botanical Laboratory 85: 103-189.

Jumpponen, A., K. Newsahan \& D.J. Nieses. 2003. Filamentous ascomycetes inhabiting the rhizoid environment of the liverwort Cephaloziella varians in Antarctica are assessed by direct PCR and cloning. Mycologia 95: 457-466.

Kreisel, H. 1971. Clave para la identificación de los macromicetos de Cuba. Ciencias Serie. 4. Ciencias 
Biológicas, Universidad de la Habana 16: 1-101.

Larraín, J. 2007. Musgos (Bryophyta) de la estación biológica Senda Darwin, Ancud, isla de Chiloé: lista de especies y claves para su identificación. Chloris Chilensis,Año $10 N^{\circ}$ 1.URL:http://www.chlorischile.cl

Lazo, W. 2001. Hongos de Chile. Atlas Micológico. Facultad de Ciencias, Universidad de Chile, 231 pp.

LePEZ, P. 1998. Estudio fitosociológico del Parque Oncol. Tesis de grado Ing. Forestal, Universidad Austral de Chile, Valdivia, Chile. 88 pp.

Llimona, X. 1997. Los Hongos. En: J. Izco y cols. (eds.), Botánica, McGraw-Hill - Interamericana de España, S.A.U., 241-308.

Minter, D.W. \& H. Peredo López. 2006. Hongos de Chile. www.cybertruffle.org.uk/chilfung [sitio Internet, versión 1.00]. Viewed: March 15, 2008.

SchaumanN, F. 2005. Terricolous bryophyte vegetation of Chilean temperate rain forests. Communities, adaptative strategies and divergence patterns. Bryophytorum Bibliotheca, Band 26. J. Cramer,
Berlin-Stuttgart, $154 \mathrm{pp}$.

Schofield, W.B. 2001. Ecology. In: W.B. Schofield (ed.), Introduction to Bryology. Segunda Edición, The Blackburn Press. 309-330.

Schuster, R.M. 2000. Austral Hepaticae. Part 1. Nova Hedwigia, Beiheft 118, 524 pp.

SмIтн, A.J.E. 1982. Epiphytes and Epiliths. In: A.J.E. Smith (ed), Bryophyte Ecology. 191-227.

UlloA, M. \& R.T. HANLIN. 2000. Illustrated Dictionary of Mycology. The American Phytopathological Society. 448 pp.

Villagrán, C., E. BarRera \& C. Medina. 2002. Las hepáticas del Archipiélago de Chiloé. CONAF, Master Print Ltda., Puerto Montt, 26 pp.

Villagrán, C., G. Hässel de Menéndez \& E. Barrera. 2005. Hepáticas y Antocerotes del Archipiélago de Chiloé. Una introducción a la flora briofítica de los ecosistemas templados lluviosos del sur de Chile. Corporación de Amigos del Museo Nacional de Historia Natural, Santiago, Chile. 160 pp.

Recibido: 22.04.08

Aceptado: 24.10 .08 